\title{
Laparoscopic treatment of profuse bleeding in Cesarean scar pregnancy
}

\author{
Markku Santala • Jatta Pirkola • Maarit Niinimäki
}

Received: 12 August 2006 / Accepted: 5 April 2007 / Published online: 5 May 2007

(C) Springer-Verlag 2007

\begin{abstract}
A case of profuse bleeding during dilation and curettage due to Cesarean scar pregnancy was treated with emergency laparoscopy. The gestational mass was removed and the perforated uterus was sutured in laparoscopy. The patient had persistent bleeding and 4 months later laparotomy was performed to explore a cystic mass in the isthmic area of the uterus. This necrotic tissue was removed and 6 months after the initial operation the patient was fully recovered with healed uterus in hysteroscopy.
\end{abstract}

\section{Keywords Cesarean scar pregnancy · Laparoscopy}

\section{Introduction}

Pregnancy in a previous Cesarean section scar is the rarest form of ectopic pregnancy and probably one of the most dangerous gestational conditions due to the risk of rupture and hemorrhage [1]. We report a patient with Cesarean scar pregnancy treated by emergency laparoscopic surgery.

\section{Case report}

A 27-year-old healthy woman was referred to our hospital for nuchal translucency examination on 17 November 2003. Her medical history consisted of one Cesarean breech delivery in 2001 and two terminations of pregnancy in 1998 and in 2002. Her early pregnancy had been uneventful. On clinical examination at 13 weeks and 4 days

M. Santala $(\bowtie) \cdot$ J. Pirkola $\cdot$ M. Niinimäki

Department of Obstetrics and Gynecology, University of Oulu, Box 5000, 90014 Oulu, Finland

e-mail: markku.santala@oulu.fi of pregnancy, the cervix was normal, but the uterus was slightly enlarged. Transvaginal sonography revealed a deformed gestational sac with solid components but no signs of fetal heart activity. There was some fluid around the uterus. Serum human chorionic gonadotropin (hCG) was not measured. The diagnosis was missed abortion.

Dilatation and curettage under general anesthesia was performed on the next day. When the cervix had been dilated to $10 \mathrm{~mm}$ and curettage initiated, profuse bleeding ensued. To identify the cause of bleeding, laparoscopy was performed. The uterus was perforated, and there were blood clots in the cul-de-sac. The gestational mass was identified, bulging in the isthmic region of the uterus (Fig. 1). The adnexal structures were normal. The gestational mass was removed and the uterine defect sutured laparoscopically using absorbable 2-0 and 3-0 synthetic absorbable sutures (Vicryl). To achieve adequate hemostasis, multiple tight sutures were required (Fig. 2). Operating time was 200 minutes. During the operation, the patient received four units of red blood cells. Total intraoperative blood loss was $3,000 \mathrm{ml}$ and the lowest hemoglobin level was $51 \mathrm{~g} / \mathrm{l}$. After the operation, the patient received a single dose of methotrexate, $50 \mathrm{mg}$ i.m. She made an uneventful recovery and was discharged from hospital on the 4th postoperative day. Serum hCG level was $567 \mathrm{IU} / 1$ on discharge.

On the 1st out-patient follow-up visit 1 month later, the patient complained of persisting scant vaginal bleeding and abdominal pain. No hCG was detected in the urine (dipstick, threshold $20 \mathrm{IU} / \mathrm{l})$. Transvaginal sonography revealed a cystic mass measuring $3.6 \times 2.6 \mathrm{~cm}$ in the isthmic region of the uterus. Two months after the operation, the patient's serum hCG level was $12 \mathrm{IU} / \mathrm{l}$. Due to the elevated serum hCG level, the persisting scant vaginal bleeding, and the transvaginal sonographic finding, magnetic resonance imaging (MRI) was performed 3 months after the laparoscopy. 


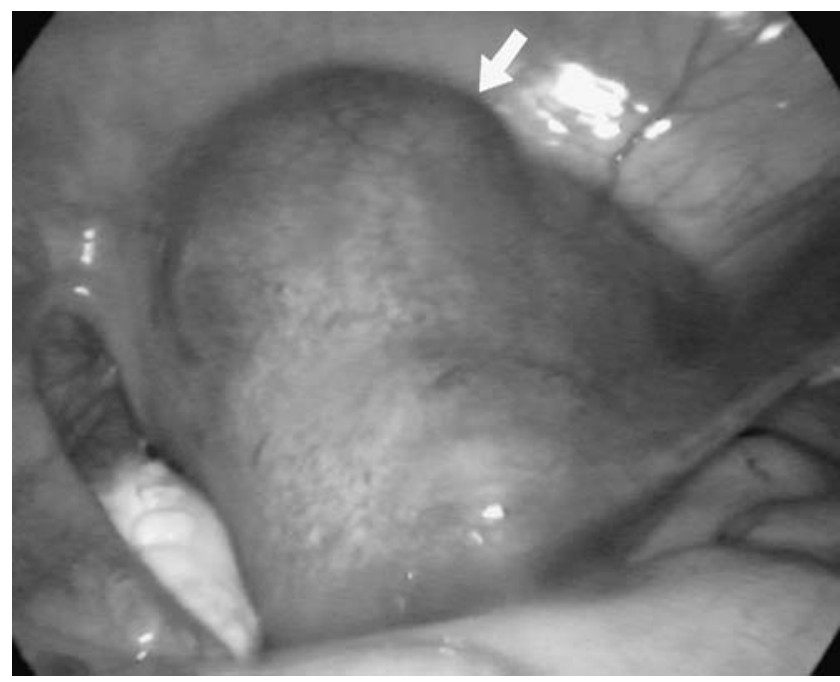

Fig. 1 Laparoscopic view of the gestational mass in the isthmic region (arrow)

MRI showed abundant vascularization in the isthmic area but did not add any information to the sonographic findings.

Elective laparotomy was performed a month later. A mass of macroscopically necrotic tissue $4 \mathrm{~cm}$ in diameter was removed from the isthmic region. Histopathologic examination showed necrotic tissue. It was impossible to identify whether the tissue was originally gestational or not. Vaginal bleeding ceased after the laparotomy. Hysteroscopic evaluation performed two months after the laparotomy revealed a healed scar covered by normal endometrium. Serum hCG level was $<5 \mathrm{IU} / \mathrm{ml}$.

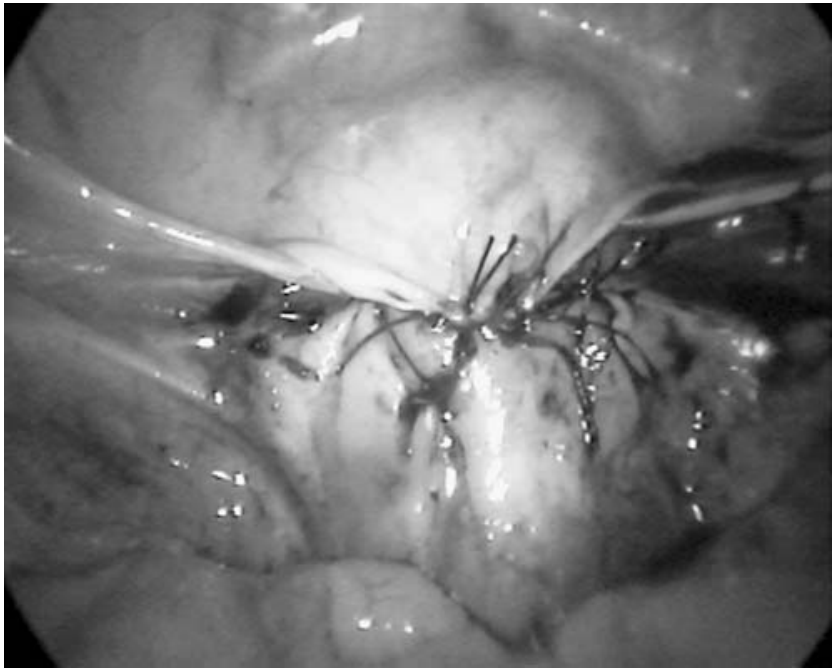

Fig. 2 Uterine defect sutured and covered with bladder peritoneum (laparoscopic view)

\section{Discussion}

Even in experienced hands, the diagnosis of Cesarean scar pregnancy can be missed in transvaginal ultrasonography. The imaging criteria are an empty uterine cavity, an empty cervical canal, development of the gestational sac in the anterior part of the uterine isthmus, and absence of healthy myometrium between the bladder and the sac [1]. Doppler sonography may show increased subtrophoblastic flow and velocity [2].The treatment of Cesarean scar pregnancy is surgical or medical. Surgical treatment aims to remove the gestational mass. We chose laparoscopy because the cause of profuse bleeding was unclear and the patient wanted to preserve her fertility. Due to the profuse uterine bleeding, we had to place many tight sutures around the uterine wound. The explanation for the persisting scant postoperative vaginal bleeding might be necrotic tissue caused by the sutures and/or remaining gestational material. It is possible that some gestational material was left in the Cesarean scar region. We chose expectant management because the hCG levels were only slightly elevated. The time required for regression of gestational material as measured by serum hCG levels and repeated transvaginal sonographic examinations is $2-12$ months [2]. Unfortunately, we had to perform laparotomy to remove the necrotic mass from the isthmic uterine region about 4 months after the laparoscopic procedure.

Women at risk for a Cesarean scar pregnancy include ones with a history of multiple Cesarean sections, Cesarean breech delivery, ectopic pregnancy, or placental pathology [3]. We suggest that a woman who has given birth by Cesarean section should be examined by transvaginal sonography early in the subsequent pregnancy if vaginal bleeding occurs. It might be prudent to consider laparoscopy combined with hysteroscopy as a treatment option if Cesarean scar pregnancy is suspected.

\section{References}

1. Fylstra D (2002) Ectopic pregnancy within a Cesarean scar: a review. Obstet Gynecol Surv 57:537-543

2. Seow KM, Huang LW, Lin YH, Lin MY-S, Tsai YL, Hwang JL (2004) Cesarean scar pregnancy: issues in management. Ultrasound Obstet Gynecol 23:247-253

3. Maymon R, Halperin R, Mendlovic S, Schneider D, Vaknin Z, Herman A, Pansky M (2004) Ectopic pregnancies in Caesarean section scars: the 8 year experience of one medical centre. Hum Reprod 19:278-284 